\title{
Predictions for the First Parker Solar Probe Encounter
}

\author{
B. van der Holst ${ }^{1}$ (1), W. B. Manchester IV ${ }^{1}$, K. G. Klein ${ }^{2}$ (1), and J. C. Kasper ${ }^{1}$ (1) \\ ${ }^{1}$ Climate and Space Sciences and Engineering, University of Michigan, Ann Arbor, MI 48109, USA \\ ${ }^{2}$ Lunar and Planetary Laboratory, University of Arizona, Tucson, AZ 85719, USA \\ Received 2019 January 29; revised 2019 February 5; accepted 2019 February 5; published 2019 February 14
}

\begin{abstract}
We examine Alfvén Wave Solar atmosphere Model (AWSoM) predictions of the first Parker Solar Probe (PSP) encounter. We focus on the 12 day closest approach centered on the first perihelion. AWSoM allows us to interpret the PSP data in the context of coronal heating via Alfvén wave turbulence. The coronal heating and acceleration is addressed via outward-propagating low-frequency Alfvén waves that are partially reflected by Alfvén speed gradients. The nonlinear interaction of these counter-propagating waves results in a turbulent energy cascade. To apportion the wave dissipation to the electron and anisotropic proton temperatures, we employ the results of the theories of linear wave damping and nonlinear stochastic heating as described by Chandran et al. We find that during the first encounter, PSP was in close proximity to the heliospheric current sheet (HCS) and in the slow wind. PSP crossed the HCS two times, at 2018 November 3 UT 01:02 and 2018 November 8 UT 19:09, with perihelion occurring on the south of side of the HCS. We predict the plasma state along the PSP trajectory, which shows a dominant proton parallel temperature causing the plasma to be firehose unstable.
\end{abstract}

Key words: interplanetary medium - magnetohydrodynamics (MHD) - methods: numerical - solar wind - Sun: corona

\section{Introduction}

The recently launched Parker Solar Probe (PSP; Fox et al. 2016) and upcoming launch of the Solar Orbiter (SO; Müller \& Cyr 2013) will provide a unique opportunity to track the evolution of structures in the inner heliosphere in unprecedented detail. PSP, with its closest perihelion of about $9.86 R_{\text {Sun }}$ from the Sun, will directly sample the solar corona in a region never before measured in situ. Solar Orbiter, with its combined suite of in situ and remote sensing instruments, will orbit within 0.28 au of the Sun, and will have opportunities to remotely observe structures in the corona and then sample those structures in situ during its near co-rotational observing periods. PSP and Solar Orbiter will revolutionize our understanding of the solar wind with a suite of instruments that will directly observe the state of the thermal plasma distributions (Kasper et al. 2016) and electric and magnetic fields (Bale et al. 2016) over a wide range of heliographic latitude and distances. These observations will detect fluid and kinetic waves, the associated turbulent cascade of energy, and signatures of wave dissipation and the consequent heating of the particle populations.

The prime PSP mission includes 24 encounters with the Sun. For each, PSP goes from 0.25 au to perihelion and back in about 11 days. The perihelion distance becomes progressively closer to the Sun as the mission progresses due to Venus gravity assists. For these orbital parameters, PSP passes very rapidly between solar wind structures at perihelion, to sub corotational speeds at aphelion. Interpreting the resultant data requires simulations for each encounter in order to put each encounter in context to understand the observations as PSP passes through both transient structures and fast and slow wind streams.

Recent advances in 3D time-dependent modeling have enabled the investigation of the evolution of the solar wind and solar transients as they escape the corona and are carried into the heliosphere (see reviews by Kilpua et al. 2017;
Manchester et al. 2017; and Gombosi et al. 2018). Extended magnetohydrodynamic (MHD) models, specifically Alfvénwave-driven models, are now capable of predicting turbulent wave amplitudes in the solar wind along with the wave reflection and dissipation rates as well as partitioning coronal heating among particle species. In addition, full kinetic models coupled to the MHD models include the effects of kinetic instabilities in the solar wind, in particular on the distribution functions of the particle species. Together the PSP and SO missions, along with state-of-the-art modeling capabilities, will provide the most powerful combination of tools to address fundamental processes in the corona and heliosphere.

At the 2018 Fall AGU meeting, several 3D global MHD model predictions of the first PSP encounter were presented. Among them were results from the CORHEL/MAS of Lionello et al. (2009), whose coronal model was recently extended to include low-frequency Alfvén wave turbulence; the ENLIL model of Odstrcil et al. (2005), which is an inner heliopshere MHD model prescribed by the empirical WangSheeley-Arge model (Arge \& Pizzo 2000); the solar corona and solar wind turbulence transport and heating model by Usmanov et al. (2018); and finally, the Alfvén Wave Solar atmosphere Model (AWSoM; van der Holst et al. 2014), which is a solar coronal and inner heliosphere model that includes low-frequency Alfvén turbulence. Here, we present the AWSoM predictions of the first PSP encounter.

\section{AWSoM Model with ADAPT-GONG Maps}

The simulations are performed with the AWSoM (van der Holst et al. 2014), which is a 3D solar corona and inner heliosphere model. AWSoM solves the single fluid magnetohydrodynamic equations extended to include proton temperature anisotropy and isotropic electron temperature. In this model, excess heat dissipated in the corona is transported back via electron heat conduction to the chromosphere where it is lost via radiative cooling. 


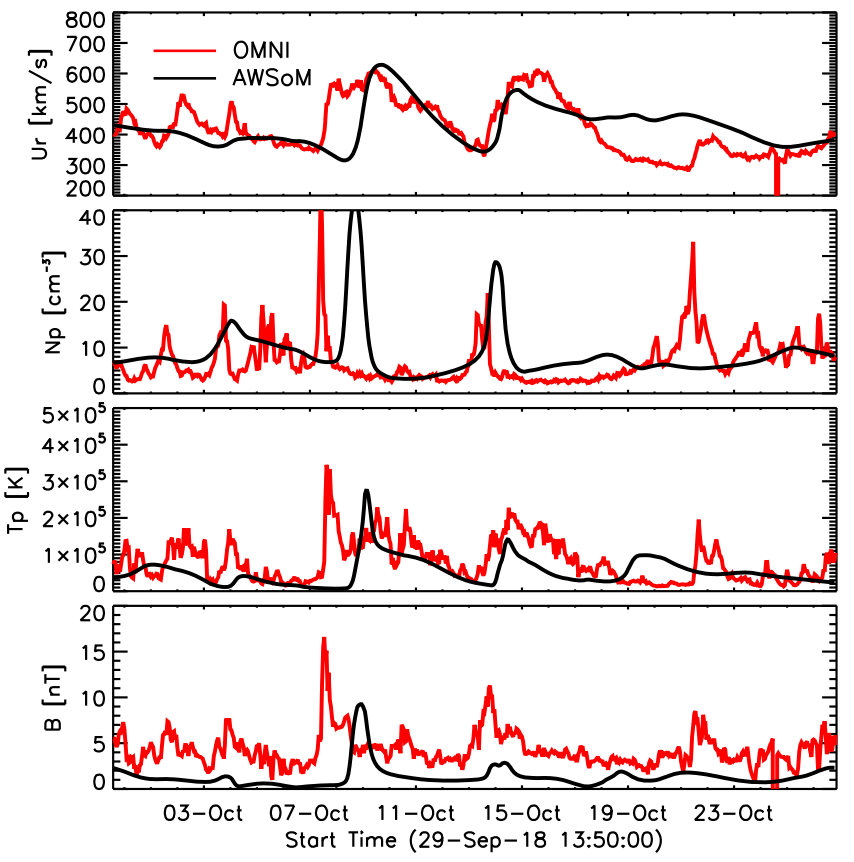

Figure 1. Comparison of the radial solar wind speed, proton number density, proton temperature, and magnetic field strength of the model output along the Earth orbit with the OMNI data for CR2209.

AWSoM addresses the coronal heating and wind acceleration via low-frequency Alfvén wave turbulence in which partial wave reflection is caused by Alfvén speed gradients. The nonlinear interaction of these counter-propagating waves results in a transverse energy cascade from the outer scale through the self-similar inertial range to the proton gyro-radius scale, where the Alfvénic cascade transitions into a kinetic Alfvén wave (KAW) cascade. Dissipation at the end of the cascade results in isotropic heating of the electrons and parallel and perpendicular proton heating. To apportion the dissipation, we include in AWSoM both stochastic ion heating by lowfrequency Alfvénic turbulence (Chandran et al. 2010) as well as linear Landau and transit time damping of KAWs, with the specific partitioning developed by Chandran et al. (2011).

Proton temperature anisotropy instabilities, specifically the Chew, Goldberger, and Low (CGL) Firehose (Chew et al. 1956), mirror (Tajiri 1967), and proton-cyclotron (Kennel \& Petschek 1966) instabilities are accounted for by adding a relaxation source term to the parallel proton pressure equation. If the plasma exceeds this stability threshold, the source term will relax the plasma back to the marginal stable state with a relaxation time that is the inverse of the growth rate of the instability.

For the predictions of the first PSP encounter, we use synchronic magnetic maps that were constructed by the Air Force Data Assimilative Photospheric flux Transport (ADAPT) model (Henney et al. 2012; Hickmann et al. 2015) based on the Global Oscillation Network Group (GONG) maps. The ADAPT model uses a flux transport model to predict the magnetic field in areas where the data are not available. ADAPT uses an ensemble of 12 model realizations based on different model parameters.

To determine which GONG-ADAPT model realization works best for the AWSoM simulation of the PSP encounter, we first performed a 1 au validation study for all 12 GONGADAPT maps for Carrington rotation (CR) 2209, which was

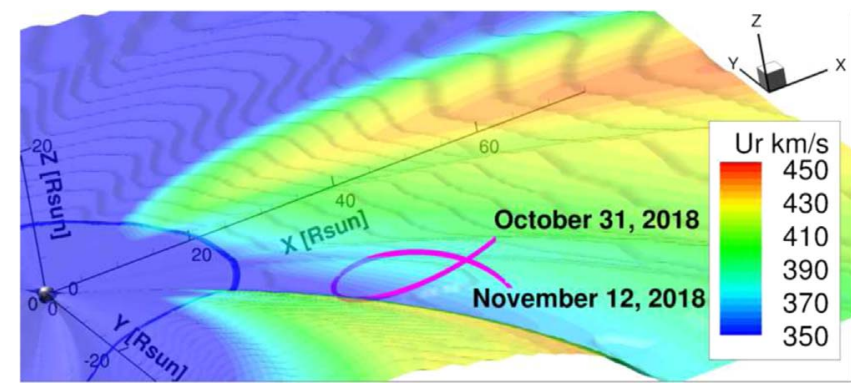

Figure 2. Heliospheric current sheet colored with radial solar wind speed. The magenta line is the PSP trajectory in the co-rotating frame. The $X, Y$, and $Z$ coordinates in the HGR system are normalized to the solar radius.

the last rotation before the PSP encounter. Figure 1 displays the solar wind speed, proton number density, proton average total temperature, and magnetic field strength along the Earth orbit for the model (black) and OMNI data (red), which contains the solar wind plasma and interplanetary magnetic field conditions at $1 \mathrm{au}$. This simulation was performed with a GONG-ADAPT map with central meridian time 2018 October 13 UT 06:00. In this figure, we only show the first realization, because for this map the model output was closest to the observed data for the last few days of CR2209.

\section{Results}

The perihelion of the first PSP encounter was at 2018 November 6 UT 3:27. To simulate the solar corona and inner heliosphere conditions for the first encounter we used the central meridian time for the GONG-ADAPT map that is closest to this time, hence we used 2018 November 6 UT 4:00. Because the first realization of the ADAPT maps provided the best 1 au results for CR2209, we used the first ADAPT realization for the PSP encounter as well. With this map we converged the AWSoM model to a steady state for the solar corona and inner heliopshere in the heliographic rotating (HGR) coordinate system.

In Figure 2, we show the heliospheric current sheet (HCS) for this steady state. This isosurface, for which the radial magnetic field is zero, is colored with the radial solar wind speed. The grayscale sphere on the left represents the Sun. The magenta line is the PSP trajectory of the first encounter from 2018 October 31 to November 12 shown in the co-rotating frame. We note that in this time frame PSP crossed according to the AWSoM simulation two times the HCS, namely 2018 November 3 UT 01:02 and 2018 November 8 UT 19:09. From this figure, we see that PSP was to the south of the HCS around the perihelion and to the north of the HCS near the beginning and end of the encounter. During the entire encounter PSP was in close proximity to the HCS.

In Figure 3, we show various plasma parameters in a section of a meridional slice through the Sun center and the PSP perihelion in the co-rotating frame. The top-left panel shows the radial velocity in color contour. Streamlines represent field lines by ignoring the out-of-plane component. The trajectory of the PSP encounter is shown as a magenta line. From this we conclude that the PSP was close to the HCS and in the slow wind. The middle-left panel shows that the proton density went to almost $500 \mathrm{~cm}^{-3}$, and the bottom-left panel shows that the radial field strength during the encounter is below $30 \mathrm{nT}$. The temperatures are shown in the right panels. During the entire encounter PSP would, according to the AWSoM model, detect 

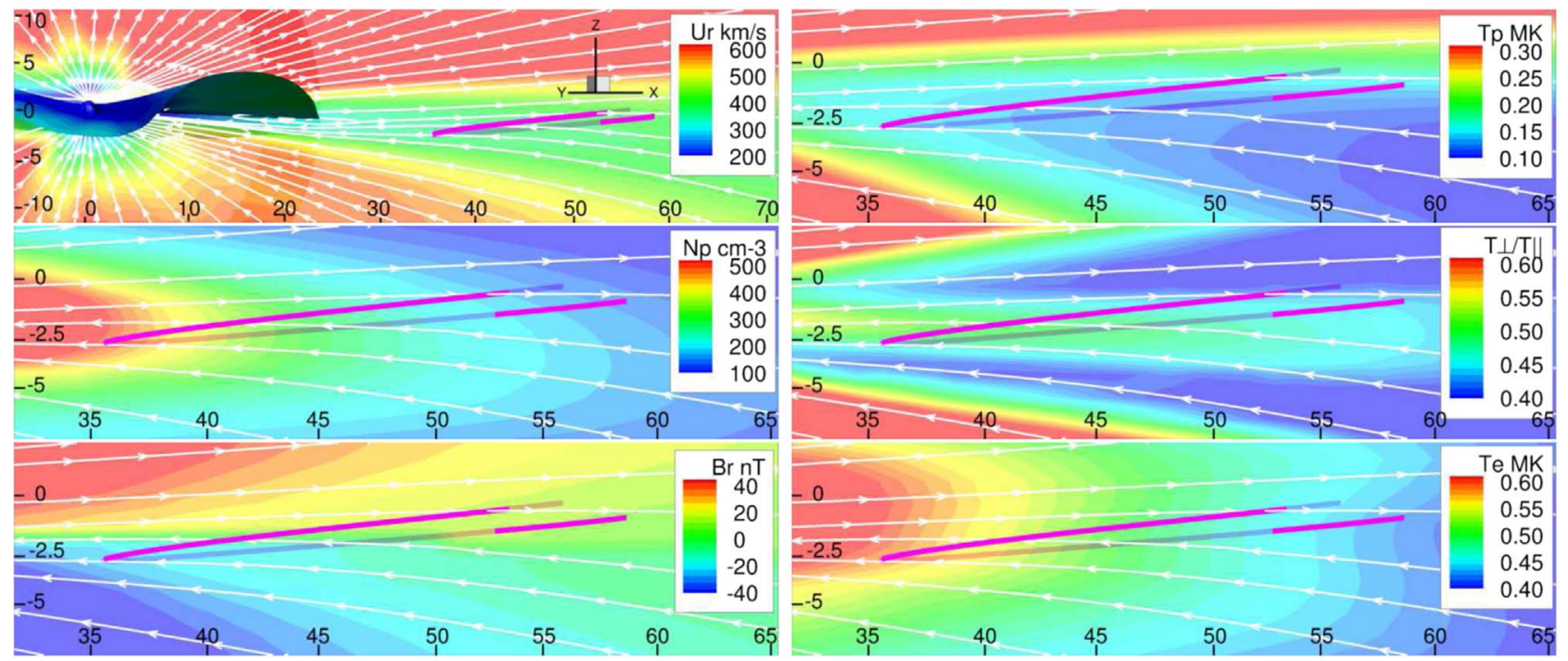

Figure 3. Plasma parameters in a meridional slice through the PSP perihelion in the co-rotating frame. Left panels (from top to bottom): radial velocity, proton number density, and radial magnetic field component in color contour. Streamlines represent field lines by ignoring the out-of-plane component. Magenta line is the PSP trajectory. The top panel shows in addition the heliospheric current sheet for $r<24 R_{\odot}$ colored with the radial velocity. Right panels (from top to bottom): averaged proton temperature, proton temperature anisotropy, and electron temperature. In all panels, the vertical axis is the Z-direction and the horizontal direction is the $R=\sqrt{X^{2}+Y^{2}}$ direction in solar radius.
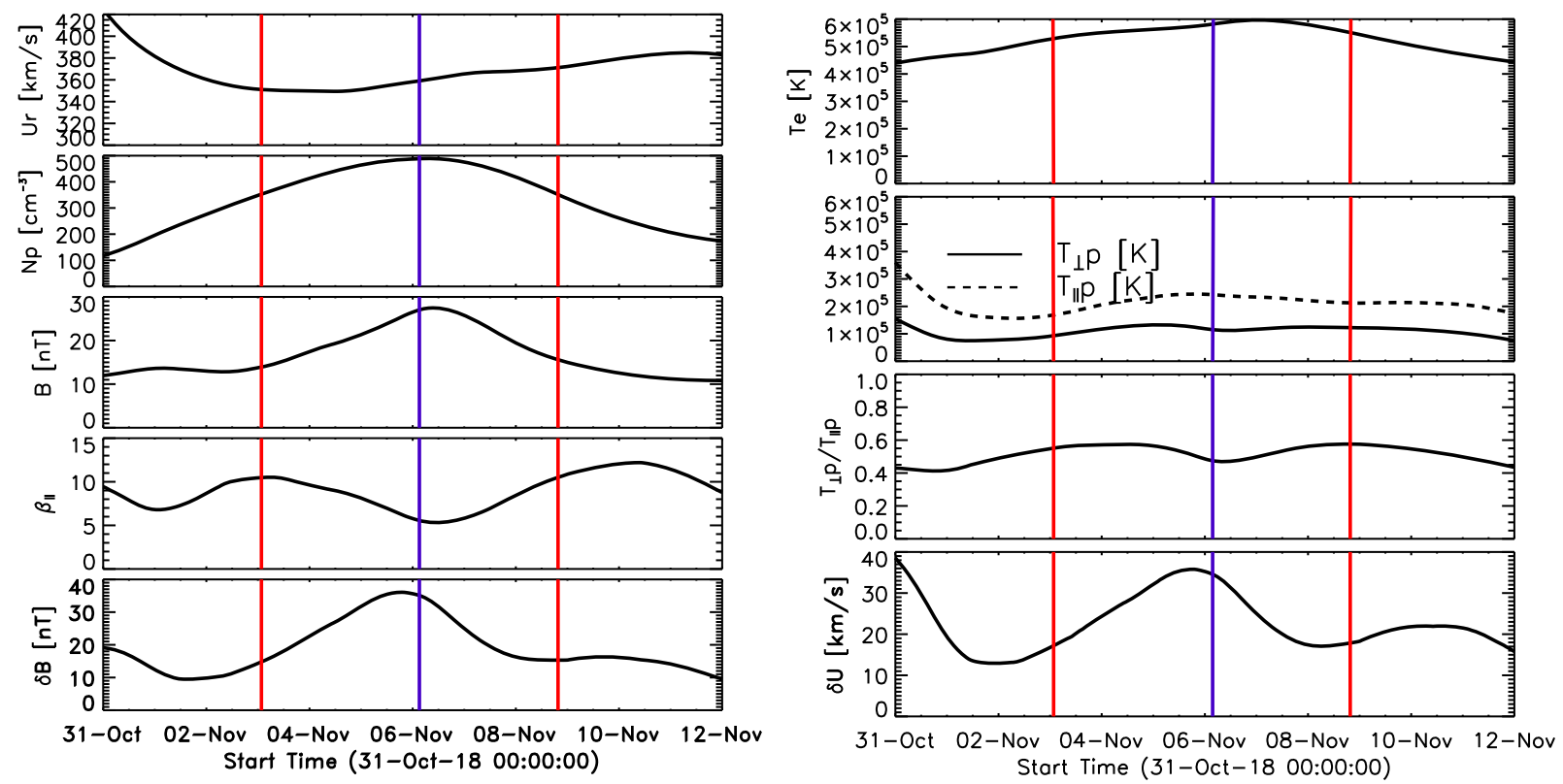

Figure 4. Simulated plasma parameters during the first PSP encounter. Left panels (from top to bottom): radial velocity, proton number density, magnetic field strength, parallel proton plasma beta $\beta_{\|}$, amplitude of Alfvén wave magnetic field fluctuation. Right panels (from top to bottom): electron temperature, parallel and perpendicular proton temperatures, proton temperature anisotropy, amplitude of Alfvén wave velocity perturbation. Blue vertical line indicates PSP perihelion, and red lines indicate heliospheric current sheet crossing.

a proton temperature anisotropy $T_{\perp} / T_{\|}$that is below 0.6 . This is a direct consequence of the significant parallel proton heating in the AWSoM model near the HCS where the plasma beta is high. Overall the average proton temperature (top-right panel) is lower near the HCS than the electron temperature (bottomright panel).

In Figure 4, we show plasma parameters as a function of time along the simulated PSP trajectory. In this figure, the PSP perihelion is indicated by a blue vertical line and the two HCS crossings are indicated by red vertical lines. The top four panels on the left are for the radial velocity, proton number density, magnetic field strength, and parallel proton plasma beta $\beta_{\| p}=2 p_{\|} \mu_{0} / B^{2}$, respectively. At perihelion, AWSoM predicts $U_{r}=360 \mathrm{~km} \mathrm{~s}^{-1}, N_{p}=490 \mathrm{~cm}^{-3}, B=27 \mathrm{nT}$, and $\beta_{\| p}=$ 5.56 for these quantities. The top three panels on the right are for the electron temperature, parallel and perpendicular proton temperature, and proton temperature anisotropy ratio (shown top to bottom). For the temperatures, AWSoM predicts at perihelion $T_{e}=0.58 \mathrm{MK}, T_{\perp p}=0.12 \mathrm{MK}$, and $T_{\| p}=$ 0.24 MK. AWSoM uses the Alfvén wave energy densities parallel and antiparallel to the magnetic field lines to calculate the impact of wave turbulence on the solar corona and inner 

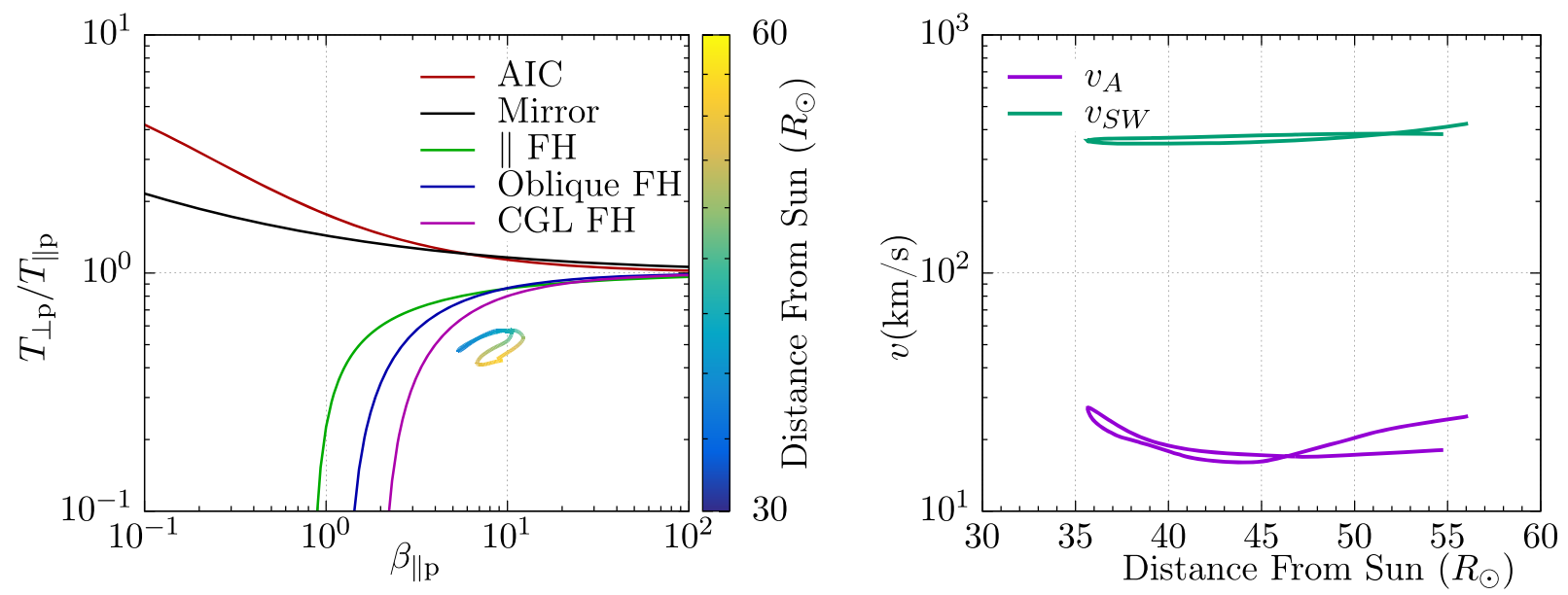

Figure 5. Left panel: PSP trajectory in the $\left(\beta_{\| p}, T_{\perp p} / T_{\| p}\right)$-plane. The plasma along the trajectory is firehose unstable. Right panel: radial solar wind speed and Alfvén speed as a function of the radial distance of PSP.

heliosphere plasma. To translate these energy densities into velocity and magnetic field fluctuations, we make an additional assumption that the reflected wave energy density propagating back to the Sun is small compared to the outward wave energy density. These fluctuations are shown in the bottom panels and we obtain at perihelion $\delta B=35 \mathrm{nT}$ and $\delta U=35 \mathrm{~km} \mathrm{~s}^{-1}$.

From the third panel on the right in Figure 4, we see that the proton temperature anisotropy $T_{\perp} / T_{\|}$stays below 0.6 along the PSP trajectory, which, together with the small magnetic field strength and hence high plasma beta, results in a firehose unstable plasma. In Figure 5, we show the trajectory in the $\left(\beta_{\| p}, T_{\perp p} / T_{\| p}\right)$-plane. Here, we also show the curves for which the proton-cyclotron, Mirror, CGL firehose, parallel firehose, and oblique firehose are marginally stable. Values for these thresholds are taken from Verscharen et al. (2016). Indeed, the PSP trajectory is located below the firehose curves, suggesting that PSP was, during the encounter, in firehose unstable plasma. We note that AWSoM currently only incorporates the CGL version of the firehose instability. The parallel and oblique firehose instabilities are planned in future updates of AWSoM and will likely result in less anisotropy in the proton temperatures. The right panel in Figure 5 shows the radial solar wind speed and Alfvén speed $\left(v_{\mathrm{A}}\right)$ versus the radial distance of PSP. The model predicts that the solar wind remains super Alfvénic over the entire trajectory of the first encounter.

\section{Summary}

In this Letter, we made predictions of the first PSP encounter with the AWSoM global solar corona and inner heliosphere model. We find that PSP was close to the HCS, the trajectory was in the slow wind, the wind speed remains super Alfvénic along the entire encounter trajectory, and the plasma along the trajectory was firehose unstable. PSP crossed the HCS two times: 2018 November 3 UT 01:02 and 2018 November 8 UT 19:09. At perihelion, PSP was to the south of the HCS. At perihelion, AWSoM predicts for the plasma state: $U_{r}=360 \mathrm{~km} \mathrm{~s}^{-1}, N_{p}=$ $490 \mathrm{~cm}^{-3}, B=27 \mathrm{nT}, T_{e}=0.58 \mathrm{MK}, T_{\perp p}=0.12 \mathrm{MK}, T_{\| p}=$ $0.24 \mathrm{MK}, \quad \beta_{\| p}=5.56, \quad v_{\mathrm{A}}=26.9 \mathrm{~km} \mathrm{~s}^{-1}, \delta B=35 \mathrm{nT}$, and $\delta U=35 \mathrm{~km} \mathrm{~s}^{-1}$. In future work, we will compare the predictions with the PSP data and make model improvements to make those comparisons better.

This work was supported by the NSF grant 1663800 and NASA grants NNX16AL12G and NNX17AI18G. The simulations were performed on the NASA Advanced Supercomputing system Pleiades. This work utilizes data produced collaboratively between AFRL/ADAPT and NSO/NISP.

\section{ORCID iDs}

B. van der Holst (i) https://orcid.org/0000-0001-5260-3944

K. G. Klein (1) https://orcid.org/0000-0001-6038-1923

J. C. Kasper (1) https://orcid.org/0000-0002-7077-930X

\section{References}

Arge, C. N., \& Pizzo, V. J. 2000, JGR, 105, 10465

Bale, S. D., Goetz, K., Harvey, P. R., et al. 2016, SSRv, 204, 49

Chandran, B. D. G., Dennis, T. J., Quataert, E., \& Bale, S. D. 2011, ApJ, 743, 197

Chandran, B. D. G., Li, B., Rogers, B. N., Quataert, E., \& Germaschewski, K. 2010, ApJ, 720, 503

Chew, G. F., Goldberger, M. L., \& Low, F. E. 1956, RSPSA, 236, 112

Fox, N. J., Velli, M. C., \& Balse, S. D. 2016, SSRv, 204, 7

Gombosi, T. I., van der Holst, B., Manchester, W. B., \& Sokolov, I. V. 2018 , LRSP, 15,4

Henney, C. J., Toussaint, W. A., White, S. M., \& Arge, C. N. 2012, SpWea, 10, S02011

Hickmann, K. S., Godinez, H. C., Henney, C. J., \& Arge, C. N. 2015, SoPh, 290, 1105

Kasper, J. C., Abiad, R., Austin, G., et al. 2016, SSRv, 204, 131

Kennel, C. F., \& Petschek, H. E. 1966, JGR, 71, 1

Kilpua, E., Koskinen, H. E. J., \& Pulkkinen, T. I. 2017, LRSP, 14, 5

Lionello, R., Linker, J. A., \& Mikić, Z. 2009, ApJ, 690, 902

Manchester, W., Kilpua, E. K. J., Liu, Y. D., Lugaz, N., Riley, P., Török, T., \& Vršnak, B. 2017, SSRv, 212, 1159

Müller, D., St., \& Cyr, O. C. 2013, Proc. SPIE, 8862, 8862

Odstrcil, D., Pizzo, V. J., \& Arge, C. N. 2005, JGR, 110, A02106

Tajiri, M. 1967, JPSJ, 22, 1482

Usmanov, A. V., Matthaeus, W. H., Goldstein, M. L., \& Chhiber, R. 2018, ApJ, 865, 25

van der Holst, B., Sokolov, I. V., Meng, X., et al. 2014, ApJ, 782, 81

Verscharen, D., Chandran, B. D. G., Klein, K. G., \& Quataert, E. 2016, ApJ, 831,128 